\title{
COMET status and plans
}

\author{
Dzmitry Shoukavy* \\ on behalf of the COMET collaboration \\ ${ }^{1}$ B.I. Stepanov Institute of Physics, National Academy of Sciences of Belarus, \\ 68 Niezaliežnasci pr., 220072 Minsk, Belarus
}

\begin{abstract}
Lepton Flavour Violation in the charged lepton sector (CLFV) is forbidden in the Standard Model. Therefore, the observation of CLFV process would be clear evidence of physics beyond the Standard Model. The COMET (COherent Muon to Electron Transitions) experiment will measure one of these processes: $\mu N \rightarrow e N$ at the Japan Proton Accelerator Research Complex in Tokai, Japan. The COMET experiment will be carried out using a two-staged approach. Phase-I of the experiment is aiming at a signal sensitivity of $3.1 \times$ $10^{-15}$. Phase-II will use much more intense beam and a more complex transport system to achieve a single-event sensitivity of $3 \times 10^{-17}$. The article gives an overview of construction and status of the COMET experiment.
\end{abstract}

\section{Introduction}

In spite of all the successes of the Standard Model (SM) related to discovery of a Higgs boson at the LHC [1],[2] it is unlikely to be the final theory of fundamental particles and their interactions that could be applicable at all energy scales. Within the framework of the SM still leaves many unanswered questions, notably: there is not any promising dark-matter candidate, no explanation for the observed matter antimatter asymmetry in the Universe, SM does not explain gravity and neutrino masses. These facts demand physics beyond the Standard Model (BSM).

The SM Lagrangian is invariant under a global $U(1)_{e} \times U(1)_{\mu} \times U(1)_{\tau}$ lepton field rotation this leads to massless neutrinos, and all lepton numbers are conserved. On other hand, lepton flavor violation (LFV) in the neutrino sector has been experimentally confirmed with the discovery of neutrino oscillations [3]. The observation of neutrino oscillations imply that neutrinos are massive, and that neutral lepton flavours are not conserved. This fact gives prerequisites for the possibility of charged lepton flavour violation (CLFV).

It should be emphasized that the phenomenon of neutrino oscillations can be explained within the framework of the SM, making its minor modification by entering the mass of the neutrino. However, in this case CLFV processes are extremely suppressed due to the small neutrino mass $\left(\mathrm{Br}<10^{-54}\right)$. So, if CLFV processes is observed at any higher rate, it will be clear evidence of BSM physics. It should be noted that BSM scenarios typically give CLFV rate much higher than SM.

This article is dedicated to to the COMET (COherent Muon to Electron Transition) experiment [4] - new high-precision experiment for searching of CLFV process through the

\footnotetext{
*e-mail: shoukavy@ifanbel.bas-net.by
} 
coherent neutrinoless conversion of a muon to an electron ( $\mu$-e conversion) in the field of an aluminium nucleus, with a single event sensitivity of $3 \times 10^{-17}$. This is a factor of $10^{4}$ better than the current limit from SINDRUM-II at PSI [5].

\section{$2 \mu-e$ conversion and background events}

\subsection{Event signature}

When a negative muon is stopped by some material, it is trapped by an atom, and a muonic atom is formed. After it cascades down energy levels in the muonic atom, the muon is bound in its $1 s$ ground state. Within the framework of the SM the following processes are possible: muon decay in orbit $\mu^{-} \rightarrow e^{-} v_{\mu} \bar{v}_{e}$ or nuclear muon capture $\mu^{-} N(A, Z) \rightarrow v_{\mu} N(A, Z-1)$. However, many BSM scenarios can lead to neutrinoless muon capture

$$
\mu^{-} N(A, Z) \rightarrow e^{-} N(A, Z)
$$

This is so called a coherent neutrinoless conversion of muons to electrons. This process violates the conservation of individual lepton flavours $L_{e}$ and $L_{\mu}$ by one unit, but the total lepton number $L=L_{e}+L_{\mu}+L_{\tau}$ is conserved. The energy of the signal electron $E_{\mu e}$ is given by:

$$
E_{\mu e}=m_{\mu}-B_{\mu}-E_{\text {recoil }},
$$

where $m_{\mu}$ is the muon mass, $B_{\mu}$ is the binding energy of the $1 s$-state muonic atom, $E_{\text {recoil }}$ is the nuclear recoil energy. Since $E_{\text {recoil }} \ll m_{\mu}, E_{\mu e}$ depends on the material in which the muon stops, for example, $E_{\mu e}$ is $104.97 \mathrm{MeV}$ for aluminium and $E_{\mu e}$ is $94.9 \mathrm{MeV}$ for lead. The COMET experiment will use an aluminium stopping target. The $\mu-e$ conversion is a very attractive process with an experimental point view. Firstly, the energy of the signal electron is above the end-point energy of the free muon decay spectrum $(\sim 52.8 \mathrm{MeV})$. Secondly, since the event signature is a mono-energetic electron, no coincidence measurement is required.

\subsection{Background events}

- Cosmic-ray background events

Cosmic rays (CR) will be a background source to $\mu-e$ conversion signal. CR muons can decay in flight or interact with the materials around the area of the muon-stopping target producing signal-like electrons in the detector region. The COMET experiment will use the Cosmic Ray Veto (CRV) system to protect the region around the detector. The CRV must identify CR muons and provide a rejection power of about $10^{-4}$ for them.

- Beam-related background events

This type of background event is caused by particles in a beam, such as electrons, pions, muons, and antiprotons. The COMET experiment will operate the proton beam with an extinction rate better than $10^{-10}$ between pulses that allow to suppress the occurrence of such background events.

- Intrinsic physics background events

Intrinsic physics background events originate mostly from muons stopped in the muon stopping target. Here, the most dangerous background is a muon decay in orbit (DIO) in a muonic atom. As previously mentioned the end-point of the Michel electron spectrum (free muon decay) about half the muon rest energy $(52.8 \mathrm{MeV})$ and far from the $E_{\mu e} \sim 105 \mathrm{MeV}$ conversion electron energy. However if the muon is bound in atomic orbit, the spectrum of electrons is 
significantly modified by their interaction with the nucleus. This leads to the endpoint of the DIO electron spectrum coincides with the energy of the signal electron $E_{\mu e}$ but with very small probability. The background can be suppressed by an ultrahigh momentum resolution of the tracker detector.

\section{COMET experiment}

The COMET experiment is seeking to measure the coherent neutrinoless conversion of a muon to an electron in the field of an aluminium nucleus, with a single event sensitivity (SES) of $3 \times 10^{-17}$ at the Japan Proton Accelerator Research Complex (J-PARC) in Tokai, Japan. Here, SES means the minimum value of the $\mu-e$ conversion rate where the experiment can still expect to observe one signal event during the running time. The COMET experiment will be carried out using a two-staged approach (see Fig.1). The first stage named as COMET
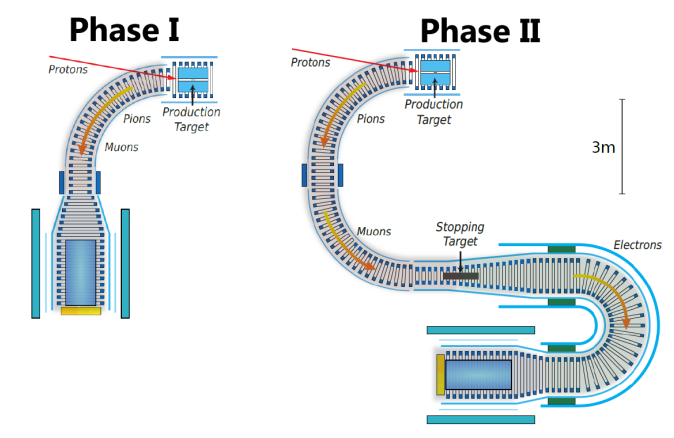

Figure 1. The schematic layout of the COMET experiment.

Phase-I is currently under construction at J-PARC. The primary goal of the COMET PhaseI is search for $\mu-e$ conversion at the intermediate sensitivity which would be 100-times better than the present limit. The second stage, COMET Phase-II is seeking another 100 improvement the usage much more intense muon beam and full length $\mathrm{C}$-shape transport solenoid. The key parameters of the COMET experiment are summarized in Table 1 and discussed in detail below.

Table 1. Summary of the Phase-I and Phase-II.

\begin{tabular}{lll}
\hline Parameter & Phase-I & Phase-II \\
\hline Beam power & $3.2 \mathrm{~kW}(8 \mathrm{GeV})$ & $56 \mathrm{~kW}(8 \mathrm{GeV})$ \\
Bending & $90^{\circ}($ beam $)+0^{\circ}($ detector $)$ & $180^{\circ}($ beam $)+180^{\circ}$ (detector) \\
Running time & $9.5 \times 10^{6} \mathrm{sec}$. & $2 \times 10^{7} \mathrm{sec}$. \\
Protons on target & $3.2 \times 10^{19}$ & $8.5 \times 10^{20}$ \\
Stopped muons on target & $1.5 \times 10^{16}$ & $2 \times 10^{18}$ \\
SES & $3.1 \times 10^{-15}$ & $2.6 \times 10^{-17}$ \\
\hline
\end{tabular}

The main idea of the COMET experiment is the usage a pulsed beam, where proton leakage between pulses is extremely reduced and make a measurements in a delayed time window. In the case of the COMET experiment, protons arrive at the primary production 
target in $100 \mathrm{~ns}$ bunches. This leads to a large flux of charged particle at the stopping target of $\sim 200 \mathrm{~ns}$. Since a muon lifetime in aluminium is $864 \mathrm{~ns}$ then a pulsed beam in the $1.17 \mu \mathrm{s}$ gap between bunches together with using delayed time window allow to eliminate of prompt beam background events.

The COMET experiment will produce muon beam from the pions decay generated in the collisions of protons with a production target made of graphite for COMET Phase-I, and a tungsten target in Phase-II. The proton beam requirements for COMET Phase-I and PhaseII differ only in their power requirements (see Table 1). They both require a pulsed of 8 $\mathrm{GeV}$ proton beam. The choice of beam energy is driven by requirement of the minimisation the production of antiprotons, production rate of which increases rapidly for proton energies above $10 \mathrm{GeV}$. Because when antiprotons reach to the detector region, they annihilate on materials releasing a large amount of energy and producing other energetic particles including pions which might produce electrons that could fake a $105 \mathrm{MeV}$ signal. The proton target will be installed within the bore of the capture solenoid and designed to maximise the capture of low energy negative pions produced in the backward direction. The production of low energy pions is of major interest since low energy muons are mostly produced by in-flight decay of them. The low energy muons can be easily stopped in an aluminium target to efficiently produce muonic atoms. Thus, the muon beamline for COMET experiment comprises the pion capture solenoid section and the muon transport solenoid section, as well as a set of matching solenoids. The curved solenoid is using for selection of the electric charge and momentum of beam particles. The center of the helical trajectory of a charged particle in curved solenoids is shifted and the drift $(\mathrm{D}[\mathrm{m}])$ is given by

$$
D=\frac{\theta_{\text {bend }}}{q B} \frac{p}{2}\left(\cos \theta+\frac{1}{\cos \theta}\right),
$$

where $q$ is the charge of the particle, $B$ is a solenoid field, $\theta_{\text {bend }}$ is the bending angle of the solenoid, $p$ is the momentum charge of the particle and $\theta=\frac{p_{T}}{p_{L}}$ is the pitch angle and $p_{L}, p_{T}$ are longitudinal and transverse momenta. The drift of the particles with the momentum of interest compensated by an auxiliary field parallel to the drift direction of

$$
B_{\text {comp }}=\frac{1}{q R} \frac{p_{0}}{2}\left(\cos \theta_{0}+\frac{1}{\cos \theta_{0}}\right),
$$

here $R$ is the the radius of curvature of the curved solenoid and in results the trajectories of charged particles with momentum $p_{0}$ and pitch angle $\theta_{0}$ are corrected.

\subsection{COMET Phase-I}

COMET Phase-I will have the pion capture section, the short muon transport section up to the end of the first $90^{\circ}$ as schematically shown on the Fig. 1. The detectors will be installed after the end of this $90^{\circ}$ bend. Phase-I will operate two different configurations. The primary COMET Phase-I detector for $\mu-e$ conversion measurement is composed of a a cylindrical drift chamber (CDC) and a set of hodoscope counters for triggering and timing, called the CyDet detector. CyDet is located after the bridge solenoid in the muon transport section, and installed inside the warm bore of a large $1 \mathrm{~T}$ superconducting Detector Solenoid. The CyDet must accurately and efficiently identify and measure $105 \mathrm{MeV}$ electrons whilst rejecting backgrounds. The muon stopping target consists of 17 aluminum disks of $0.2 \mathrm{~mm}$ thickness and $100 \mathrm{~mm}$ radius with $50 \mathrm{~mm}$ spacing is placed in the center of the CyDet, where muons are captured and $\mu-e$ conversion can be occur. The muon yield per proton obtained from simulation is $4.7 \times 10^{-4}$ and the fraction of captured muon to the total muons on target 
is 0.61 . Thus, to achieve the the design single event sensitivity of COMET Phase-I a total number of protons on target (POT) of $3.2 \times 10^{19}$ i.e $1.5 \times 10^{16}$ stopped muons on target which corresponds to about 150 days is required (see Table 1 ).

The second configuration has an aim to make direct measurement of potential background for the full COMET experiment using prototypes of the Phase-II straw tracker and the electron calorimeter (ECAL), called StrEcal detector. Prototype version of the StrEcal detector will be install in vacuum and magnetic field of $1 \mathrm{~T}$ in place of CyDet. The straw tracker for Phase-I consists of five stations, but possibly more for Phase-II. Each station has four planes; two to measure the $x$ coordinate and two to measure the $y$ coordinate. The straw tube for Phase-I are $9.8 \mathrm{~mm}$ in diameter and $20 \mu \mathrm{m}$ in wall thickness made by an aluminised-Mylar. The operating gas mixture is $50 \% \mathrm{Ar}-50 \% \mathrm{C}_{2} \mathrm{H}_{6}$. The specifications for ECAL are determined by its requirements for Phase-II running, which are an energy resolution of better than $5 \%$ at $105 \mathrm{MeV}$ and a cluster position resolution that is better than $1 \mathrm{~cm}$. It will also provide an additional hit position on the electron track trajectory and provide the trigger signals. The ECAL will be built of LYSO crystals which have a $2 \times 2 \mathrm{~cm}^{2}$ cross-section and whose length is $12 \mathrm{~cm}$, corresponding to 10.5 radiation lengths. The basic unit of the ECAL is a $2 \times 2$ crystal module. The StrEcal detector will be regarded as a final prototype of the COMET Phase-II detector.

\subsection{COMET Phase-II}

Phase-II will use the complete C-shaped muon transport solenoid with bend of $180^{\circ}$ accordingly the accuracy of the selection of particles for electric charge and momentum is improved. COMET Phase-II also has an additional 180。 curved solenoids which is used to discard electrons that are not of interest (see Fig.1). It allows to transport the signal electrons from the muon stopping target to the detector section with high efficiency.

The straws for COMET Phase-II are $5 \mathrm{~mm}$ in diameter and $12 \mu \mathrm{m}$ in wall thickness to reach a momentum resolution of better than $150 \mathrm{keV} / \mathrm{c}$. It allows to have enough momentum resolution of electron measurement and the signal and DIO background separation will be clear. During Phase-II the ECAL covers the cross-section of the $50 \mathrm{~cm}$ radius detector region and 1920 crystals are needed. The calorimeter is located behind the straw tracker and provides a fast trigger signal if the deposited energy lies within a given range.

It should be noted that the additional improvement of the the design single event sensitivity of COMET Phase-II specified in Table 1 by one order of magnitude using the same beam power are being considered. This improvement requires the optimization of the design of the proton and muon targets as well as the electron spectrometer.

\section{COMET preparation status}

The COMET experiment preparation is intensively in progress. Construction of the COMET building is completed (see Fig.3). The COMET Phase-I transport solenoid with a bending angle of $90 \circ$ is already installed in the COMET hall as shown on Fig.4. The CDC is used for the Phase-I physics measurement is constructed. Cosmic Ray test of the CDC at KEK is done. The spatial resolution of $170 \mu \mathrm{m}$ at is obtained. The mass production of 2500 straw tubes for Phase-I is finished. The test of the StrECAL protopype was successfully done, the trigger signal was provided by ECAL, and the electron track was successfully reconstructed by straw tubes. The energy resolution of ECAL of $4.4 \%$ at $105 \mathrm{MeV}$ and the position resolution of the straw tracker less than $150 \mu \mathrm{m}$ were obtained that fulfill the requirements. 


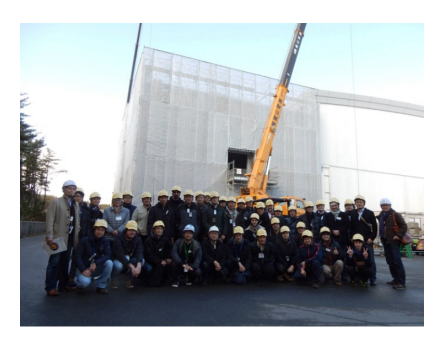

Figure 2. The COMET collaboration in front of the COMET building in January 2015.

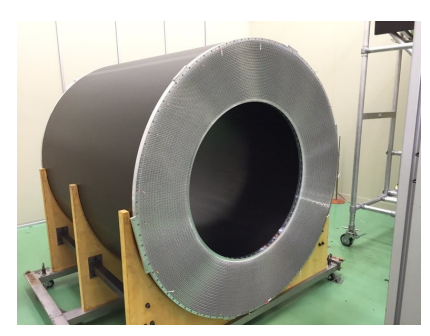

Figure 4. The construction of CDC was successfully completed.

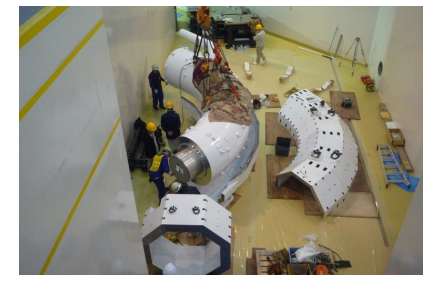

Figure 3. An installation of the transport solenoid in the COMET hall

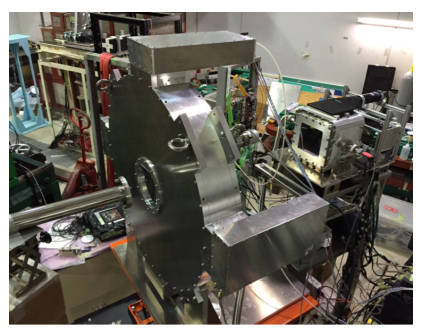

Figure 5. The test of the StrECAL prototype at Tohoku university.

\section{Conclusion}

CLFV rate in the SM with non-zero neutrino mass is small to be observed in experiments. Therefore, any signal is a clear indication of physics beyond of the Standard Model. COMET experiment search is a new high-precision experiment for searching of CLFV process through the coherent neutrinoless conversion of a muon to an electron in the field of an aluminium nucleus, with a single event sensitivity by at least four orders of magnitude over than current limit. The COMET Collaboration will have its detector systems commissioned and tested by the end of JFY 2019, to be ready for the beam which will arrive subsequently. Beam studies in the "B-line" proton beam line which supplies COMET will commence at this time. The full COMET Phase-II will be deployed and running after the completion of Phase-I experiment.

\section{Acknowledgement}

We acknowledge support from JSPS, Japan; Belarus; NSFC, China; IHEP, China; IN2P3CNRS, France; CC-IN2P3, France; SRNSF, Georgia; DFG, Germany; JINR; IBS, Korea; RFBR, Russia; STFC, United Kingdom; and Royal Society, United Kingdom

\section{References}

[1] G. Aad et al. (ATLAS), Phys. Lett. B716, 1 (2012), 1207. 7214

[2] S. Chatrchyan et al. (CMS), Phys. Lett. B716, 30 (2012), 1207. 7235

[3] Y. Fukuda et al. (Super-Kamiokande), Phys. Rev. Lett. 81, 1562 (1998), hep-ex/9807003

[4] G. Adamov et al. (COMET) (2018), 1812.09018

[5] W.H. Bertl et al. (SINDRUM II), Eur. Phys. J. C47, 337 (2006) 